\title{
Optimization of Contact Edge Profile for Minimizing Contact Pressure in a Press-fitted Shaft
}

\author{
Dong-Hyung Lee ${ }^{1, *}$, Ha-Young $\mathrm{Choi}^{2}$, Seok-Jin Kwon ${ }^{1}$, and Jeong-Won Seo ${ }^{1}$ \\ ${ }^{1}$ Fatigue and Fracture Research Team, Korea Railroad Research Institute, Korea \\ ${ }^{2}$ Department of Mechanical Engineering, Dongyang Mirae University, Korea
}

\begin{abstract}
In the shrink or press-fitted shafts such as railway axles, the rotor of a steam turbine or coupling, a high-stress concentration takes place in the close of contact edge due to relative slip between shaft and boss in a press-fitted shaft and this is a major cause of fatigue failure of the shaft. The object of this paper is to build a finite element analysis model for analysing press-fitted and bending load condition in a pressfitted assembly and is to propose a hub shape optimization method to minimize a contact pressure in the close of shaft contact edge. Numerical asymmetric-axisymmetric finite element model was developed to predict the contact stress state of the press-fitted shaft. Global optimization method, genetic algorithm, and local optimization method, sequential quadratic programming, was applied to the press-fitted assembly to optimize the hub contact edge geometry. The results showed that the maximum contact pressure with optimized hub shape decreased more than $60 \%$ compared to conventional hub shape, the maximum contact stress affecting fatigue life reduced about $47 \%$. In addition, hub shape optimization design could be a useful tool, able to increase the load capabilities of press fits concerning wear and fatigue behaviour.
\end{abstract}

\section{Introduction}

The wheelset of the railway vehicle is a structure in which an axle and a wheel are press-fitted, and fretting damage occurs at the end of a fitted region between the axle and the wheel. Fatigue strength of press-fitted shaft is reduced by $50 \%$ compared to normal fatigue strength due to fretting occurring at the axle [1]. The study on the improving fatigue strength of press-fitted shaft can be distinguished mainly into three categories such as a study on the design effects of the press-fitted shaft edge, a study on the effect of the residual stress of shaft, and a study on the damage reduction of fretting [1]. The study on the effect of residual stress is to improve fatigue strength by generating compressive residual stress on the shaft through carburizing or induction heat treatment. The research on the reduction of fretting damage is about preventing or reducing fretting corrosion through coating contact surface or changing the material properties of the contact part.

There are some studies on the design effects of the pressfitted shaft edge. Peterson and Wahl [2] represented creating a groove to relieve stress concentration at the end of the hub is useful for fatigue strength improvement through the photoelastic experiment. Nishioka and Komatsu [3] tested different shape for the pressed-fitted shaft structures and showed that fatigue limit with overhanging hub structure is increased by $50 \%$. Kubota et al. [4] reported that proper grooving of the shaft is effective for improving the fretting fatigue limit. Lee [5] applied a taper to the end of hub contact edge and reported that the appropriate taper is effective in improving the fatigue life. Biron [6] proposed an optimization method for an overhang type press-fitted shaft, which minimizes the weight and maximizes the fatigue life of the contact and fillet parts. The above series of studies to improve fatigue strength in the pressfitted shaft contributed significantly to increase the fatigue life on the axle. However, making grooves on the shaft may cause the crack in the groove area, and the fatigue life is decreased. In addition, in case of the axle, a study is required to improve fatigue strength without significantly changing the shape of the shaft or hub during the maintenance phase.

Therefore, in this study, the contact edge shape of the hub is optimized to minimize contact pressure, which directly affects the fatigue life, through the finite element analysis and the numerical analysis for the press-fitted shaft. FEA model of the press-fitted shaft subjected to fatigue bending loads has been built to analyse contact pressure and contact stress. Using the FEA results and the approximate optimization method, the optimal hub design that minimizes contact pressure of the contact edge of a press-fitted shaft is derived. The effectiveness of the optimization procedure is verified by comparing contact pressures and stresses of the press-fitted shaft with the optimized hub shape and traditional hub shape.

\section{Analysis methods}

\subsection{Finite element analysis}

* Corresponding author: dhlee@,krri.re.kr 
The analysis model is for the press-fitted test specimen subjected to the fretting fatigue test, and the structure and shape of the specimen are shown in Fig. 1. In order to calculate the stress distribution of the press fit and the loaded condition, we used a commercial FE code ABAQUS [7], which is widely used in non-linear numerical analyses.

The press-fitted specimen is modelled using an asymmetric-axisymmetric element (CAXA4R4), and for the details of the modelling method, we refer the reader to the authors' previous report [5]. As the stress distribution at the end of contact area changes quickly due to micro-slip, the minimum element at the contact end is divided by $0.015 \mathrm{~mm}$ as shown in Fig. 2 . Shrinkage allowance on the contact surface ensures that the Lamé's nominal contact pressure [8] is $90 \mathrm{MPa}$. The nominal bending stress of $135 \mathrm{MPa}$, which is fatigue limit of the press-fitted shaft specimen, is applied, and for the friction interpretation, Lagrange multiplier friction formulation and 0.6 for the coefficient of friction are used.

\subsection{Shape optimum design method}

When the load is applied to the press-fitted shaft, the contact pressure is calculated by FEA, and the approximate model for optimization is constructed. Approximate optimization has the advantage of reduction of time consumption compared to the optimization method through the numerical analysis or experiment directly. The approximate model is created

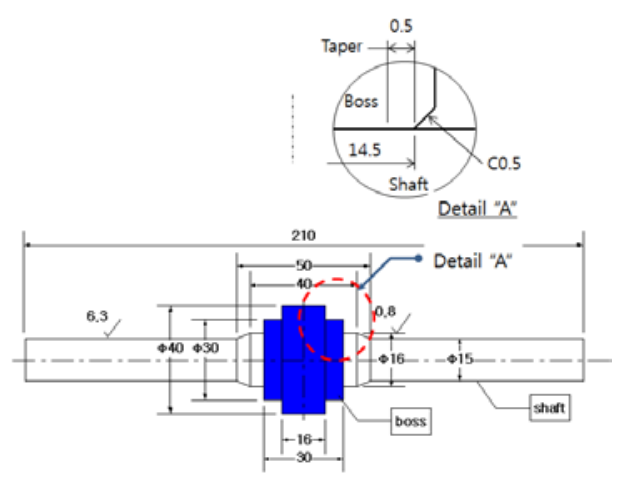

Fig. 1 Geometry of the press-fitted fatigue specimen.

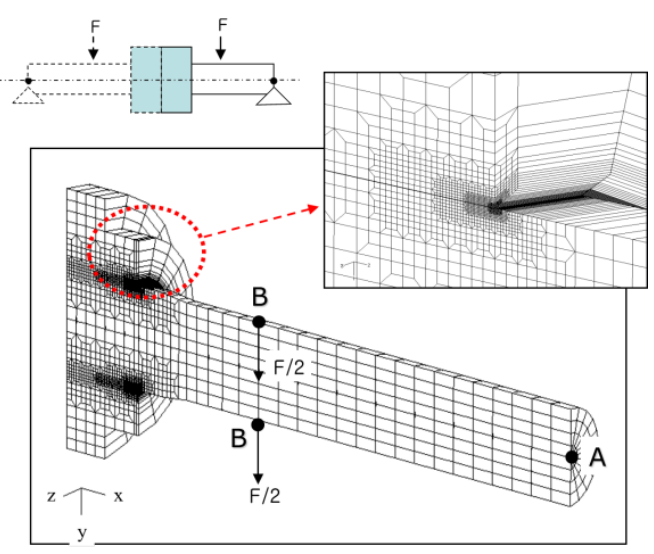

Fig. 2 Finite element model for press-fitted specimen. by the Response Surface Method (RSM) [9], and as the optimization method, Genetic Algorithm (GA) [10] that is global optimization method and Sequential Quadratic Programming (SQP) [11] that is local optimization method, is applied.

Fig. 3 represents the optimization procedure of hub shape design. The analytical samples are extracted by Latin Hypercube Sampling (LHS), and the values of contact pressure along the contact surface are obtained from the finite element analysis according to the change of contact edge shape. After generating the approximate model using the response surface method, the optimum design is performed through the generated approximate model and optimization algorithms. The objective function of the optimization is defined as the contact pressure along the contact surface of the press-fitted shaft, and the design variables are formulated as shown in Fig. 4. In Fig. 4, the location of $p_{2}$ is the end of the hub, and the location of $p_{0}$ is the inner region of the hub at a distance $0.5 \mathrm{~mm}$ from the end of the hub. Because fatigue damage occurs at $0.2 \sim 0.3 \mathrm{~mm}$ inner region from the end of contact edge, the variable, $p_{0}$, is set to 0.5 $\mathrm{mm}$.

According to the result of the previous study [5], the magnitude and location of the maximum contact pressure vary by the amount of taper at the end of

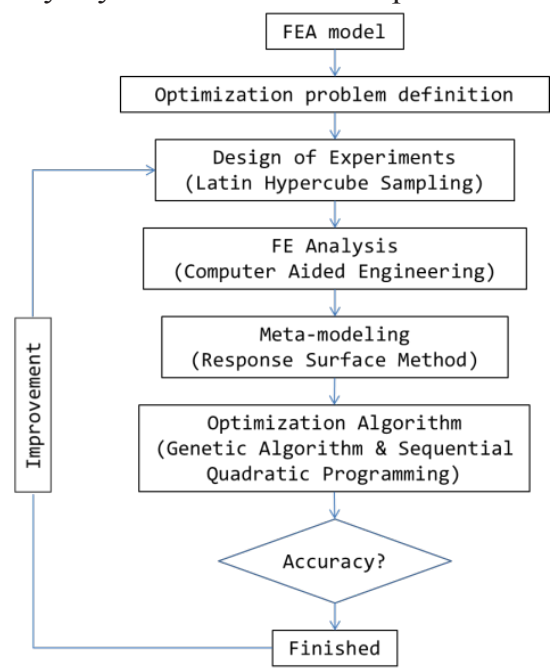

Fig. 3 Workflow of optimization process.

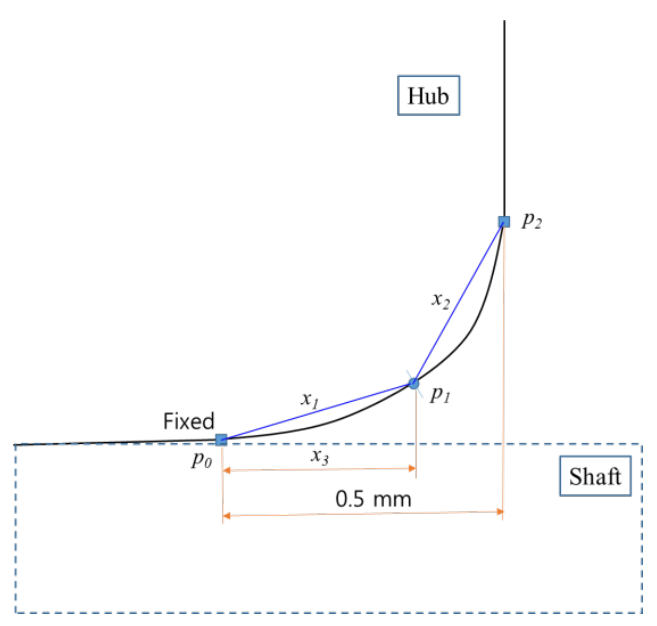

Fig. 4 Design variables of hub edge profile. 
contact edge, so the inclination of hub contact edge is set as a variable. The two gradients, $x_{1}$ and $x_{2}$, and the horizontal coordinates of the location where gradient changes, $x_{3}$, are defined as design variables. The inner contact profile of the hub connecting the three points $p_{1}$, $p_{2}$ and $p_{0}$ is created with curve by using Piecewise Cubic Hermite Interpolating Polynomial (PCHIP). The location $p_{1}$ is located between $p_{0}$ and $p_{2}$, and the gradient of the two points, $p_{1}$ and $p_{0}$, is $x_{1}$, and the gradient of the two points, $p_{1}$ and $p_{2}$, is $x_{2}$. The final formulation for optimization is defined as follows:

Minimize $\mathrm{F}_{\text {contact pressure }}$

Design variables

$$
\begin{aligned}
& 0.000 \leq x_{1} \leq 0.010 \\
& 0.010 \leq x_{2} \leq 0.015 \\
& 0.000 \leq x_{3} \leq 0.450
\end{aligned}
$$

The approximate model generation for the optimization problem of this study uses the experimental planning method, Latin Hypercube Sampling, which is widely used in the field of engineering. The approximate model equation is established using the Response Surface Method with Latin Hypercube Sampling and Finite Element Analysis, and its accuracy is shown in Fig. 5.

\section{Results and reviews}

\subsection{Finite element analysis results}

Fig. 6 shows the analysis results of the distribution of contact pressure at the end of the shaft contact edge when the compressive load and no load are applied to the press-fitted shaft. In this figure, the $\mathrm{x}$ - and $\mathrm{y}$-axes show the normalized values through the $y$-axis is divided by the nominal contact pressure $(90 \mathrm{MPa})$ and the $\mathrm{x}$-axis is divided by the contact length ' $a$ ' $(14.5 \mathrm{~mm})$. As shown in the figure, even when no load is applied to the pressfitted shaft, the contact pressure at the end of contact edge is approximately four times higher than the nominal contact pressure because of press fitting. When the bending load is applied to the press-fitted shaft, the maximum contact pressure is higher than that of pressfitted state (no load) and is approximately 11 times of the nominal contact pressure. Therefore, the significant contact pressure is generated at the end of the contact

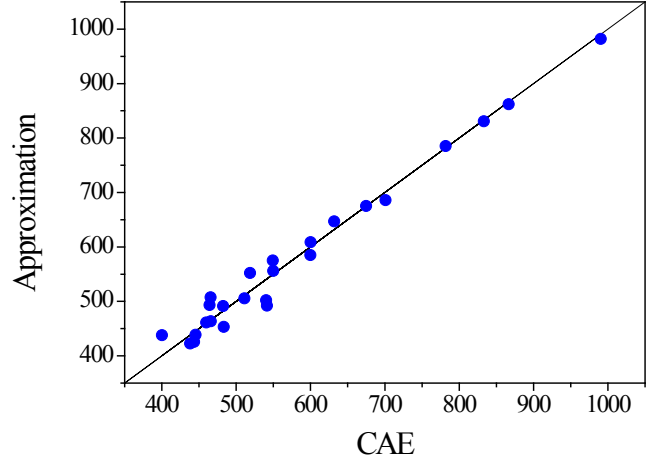

Fig. 5 Accuracy of real CAE and RSM results.

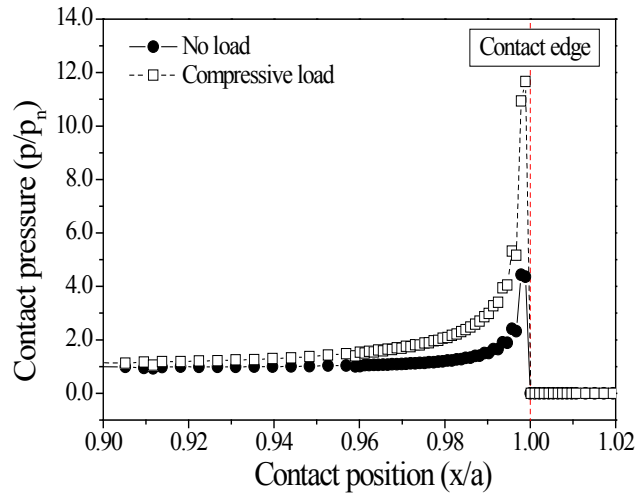

Fig. 6 Contact pressure distributions of press-fitted shaft.

edge and it can be seen that the stress concentration in this region causes the fretting damage.

\subsection{Optimization results}

The results of optimizing the hub design of the pressfitted shaft using genetic algorithms and sequential quadratic programming are shown in Table 1.

With the approximate model that is created by response surface method, the optimization with the genetic algorithm is performed by setting parameters as follow; the number of generations to 100 , number of population of 100 , mutation rate of $30 \%$, and crossover rate of $10 \%$. Since the SQP algorithm is influenced by the position of the initial design variables, the initial design variables are set to three points.

The optimal and lowest maximum contact pressure is

\begin{tabular}{|c|c|c|c|c|c|c|}
\hline & & \multirow{2}{*}{ Original } & \multicolumn{4}{|c|}{ Optimization } \\
\hline & & & GA & SQP-1 & SQP-2 & SQP-3 \\
\hline \multicolumn{2}{|c|}{$\mathrm{x}_{1}\left(\mathrm{x} 10^{-3} \mathrm{~mm}\right)$} & - & 3.41 & 3.43 & 3.39 & 3.38 \\
\hline \multicolumn{2}{|c|}{$\mathrm{x}_{2}\left(\mathrm{x} 10^{-3} \mathrm{~mm}\right)$} & - & 12.59 & 12.55 & 12.45 & 12.53 \\
\hline \multicolumn{2}{|c|}{$\mathrm{x}_{3}\left(\times 10^{-3} \mathrm{~mm}\right)$} & - & 269.85 & 273.39 & 270.73 & 267.14 \\
\hline \multirow{2}{*}{$\begin{array}{c}\text { Contact } \\
\text { pressure } \\
(\mathrm{MPa})\end{array}$} & CAE & 1,050 & 393.5 & 398.6 & 399.6 & 393.3 \\
\hline & Approx. & - & 371.8 & 372.0 & 371.9 & 371.9 \\
\hline \multicolumn{2}{|c|}{$\begin{array}{c}\text { Initial Values } \\
\left(\times 10^{-3} \mathrm{~mm}\right)\end{array}$} & - & - & $\begin{array}{c}\mathrm{x}_{1}: 2.50 \\
\mathrm{x}_{2}: 11.25 \\
\mathrm{x}_{3}: 112.50\end{array}$ & $\begin{array}{c}\mathrm{x}_{1}: 5.00 \\
\mathrm{x}_{2}: 12.50 \\
\mathrm{x}_{3}: 225.00\end{array}$ & $\begin{array}{c}\mathrm{x}_{1}: 7.50 \\
\mathrm{x}_{2}: 13.85 \\
\mathrm{x}_{3}: 337.50\end{array}$ \\
\hline
\end{tabular}
obtained from the analysis case of SQP-3 and the

Table 1. Optimization results 
maximum contact pressure is reduced by $63 \%$ from that of original design in compressive loading condition. The approximate value of the maximum contact pressure derived from the optimized design is $371.9 \mathrm{MPa}$ and the value of finite element analysis is $393.3 \mathrm{MPa}$, and there is $6 \%$ in error of contact pressure.

The results of the SQP with three initial values have almost same values with those of the optimization with GA. The program running time with SQP is about 60 times shorter than that with the genetic algorithm, and for this optimal design, it is clear that the performing the optimization with SQP is more efficient than that with GA.

Fig. 7 and Fig. 8 shows the distribution of contact stress (von Mises) on the shaft contact edge when the compressive load is applied to the normal and optimal press-fitted assembly, respectively. For normal pressfitted assembly, high stress up to about $1,000 \mathrm{MPa}$ is produced at the end of contact edge of the shaft. For the optimized press-fitted assembly, due to the curved inner geometry of hub, a maximum stress of $530 \mathrm{MPa}$ is occurred at $0.25 \mathrm{~mm}$ position inside the shaft-hub contact end, and the maximum stress is reduced by about $47 \%$ compared with the conventional one. From these results, it can be concluded that the fatigue life of optimal designed press-fitted shafts is greatly increased, which is effective in reducing the fretting damage.

\section{Conclusions}

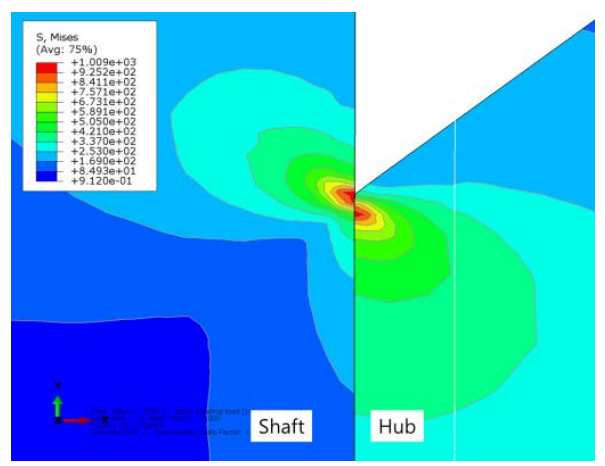

Fig. 7 Contact stress (von Mises) distribution of conventional press-fitted assembly.

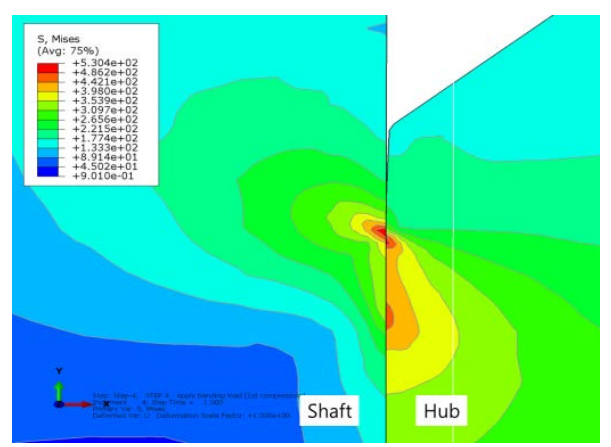

Fig. 8 Contact stress (von Mises) distribution of optimized press-fitted assembly.
A finite element analysis model is constructed for a press-fitted shaft, which is widely used in general industrial machinery, and the optimal design method of hub shape is proposed by using the approximation optimization method.

As a result of applying the genetic algorithm and the sequential quadratic programming to optimize the shape of the press-fitted shaft, there is no difference in the optimization results, and the analysis time with SQP algorithm was much shorter than that with the genetic algorithm.

The contact pressure of the optimized press-fitted shaft decreased by about $60 \%$ compared to the normal one, and the contact stress that affects the fatigue life is reduced by about $47 \%$. From these results, it can be concluded that the proposed hub shape optimization method is effective for improving fatigue strength in a press-fitted assembly.

This research was supported by a grant from R\&D Program of the Korea Railroad Research Institute, Republic of Korea.

\section{References}

1. Hirakawa, K., Toyama, K. and Kubota, M., "The Analysis and Prevention of Failure in Railway Axles," Int. J. Fatigue, Vol. 20, No. 2, pp. 135 144, 1998.

2. Peterson, R.E., and Wahl, A.M., "Fatigue of shafts at fitted members with a related photoelastic analysis," J Appl Mech, Vol. 2, No. 1, pp.A1-A11, 1935.

3. Nishioka, K. and Komatsu, H., "Researches on increasing the fatigue strength of press-fitted shaft assembly,” Bull JSME, Vol. 10, pp.880-889, 1967.

4. Kubota, M., Kataoka, S. and Kondo, Y., "Effect of stress relief groove on fretting fatigue strength and index for the selection of optimal groove shape," Int. J. of Fatigue, V. 31, Issue 3, pp. 439-446, 2009.

5. Lee, D.H., Kwon, S.J., Seo, J.W., Kwon, S.T. and You, W.H., "Evaluation of Fatigue Crack Initiation Life according to the Hub Contact Shape in a Pressfitted Shaft," KSPE Spring conference, 2010.

6. Biron, G., Vadean, A. and Tudose, L., "Optimal design of interference fit assemblies subjected to fatigue loads," Struct Multidisc Optim, 47, pp. 441451, 2013.

7. Abaqus ${ }^{\circledR}$ Version 6.10 Analysis User's Manual, Dassault Systèmes, 2010.

8. Ugural, A.C. and Fenster, S.K., "Advanced strength and applied elasticity,” Prentice-Hall, Inc. 1995.

9. Hong, K. J., Jeon, K. K., Cho. Y. S., Choi, D. H., Lee, S. J., "A Study on the Construction of Response Surface for Design Optimization," Trans. of the KSME(A), 24(6), 1408-1418, 2000.

10. Holland, J.H., "Adaptation in natural and artificial systems," Ann Arbor, University of Michigan Press, 1975. 
11. Fletcher, R., "Practical methods of optimization," John Wiley and Sons, 1987.

12. Simpson, T. W., Lin, D. K. J., Chen, W., "Sampling Strategies for Computer Experiments: Design and Analysis," International Journal of Reliability and Applications, 2(3), 209-240, 2001.

13. Fritsch, F. N. and Carlson, R. E., "Monotone Piecewise Cubic Interpolation," SIAM J. Numerical Analysis, Vol. 17, No. 2, pp. 238-246, 1980. 